\title{
Capacidad predictiva de la inteligencia emocional sobre el apoyo social percibido de adolescentes
}

\author{
Mercedes Nancy Jiménez Rosario ${ }^{a *}$, Inge Axpe Sáez e lgor Esnaola ${ }^{\mathrm{b}}$ \\ ${ }^{a}$ Universidad del País Vasco/Euskal Herriko Unibertsitatea (UPV/EHU), Vizcaya, España \\ ${ }^{b}$ Universidad del País Vasco/Euskal Herriko Unibertsitatea (UPV/EHU), Gipuzkoa, España
}

Recibido el 4 de febrero de 2020; aceptado el 10 de junio de 2020

\section{PALABRAS CLAVE}

Inteligencia emocional, apoyo familiar, apoyo de los amigos, apoyo de personas significativas, adolescencia

\begin{abstract}
Resumen Por medio del presente estudio se busca analizar la capacidad predictiva de la inteligencia emocional medida tanto desde el modelo de habilidad (atención, claridad y reparación emocional) como desde un modelo mixto (intrapersonal, interpersonal, manejo del estrés y adaptabilidad). Esto, sobre cada una de las fuentes del apoyo social percibido (familiares, amigos y personas significativas), teniendo en cuenta el sexo y la edad de los adolescentes. La muestra estuvo compuesta por 1030 estudiantes de educación secundaria con edades entre 11 y 19 años residentes en la República Dominicana. Los resultados señalan que la inteligencia emocional predice la percepción del apoyo social en las muestras de ambos sexos, aunque entre las chicas el efecto explicativo es mayor que entre los varones. Se observa también que la capacidad predictiva es mayor en la adolescencia media. Estos datos refuerzan la necesidad de desarrollar la inteligencia emocional.
\end{abstract}

(C) 2020 Fundación Universitaria Konrad Lorenz. Este es un artículo Open Access bajo la licencia CC BY-NC-ND (http://creativecommons.org/licenses/bync-nd/4.0/).

Predictive capacity of emotional intelligence on perceived social support of adolescents

Abstract Through this study we seek to analyze the predictive capacity of emotional intelligence measured, both from a model of skills (attention, clarity and emotional repair) and a mixed model (intrapersonal, interpersonal, stress management and adaptability), on each of the sources of perceived social support (family, friends and significant people), according to sex and age. The sample consisted of 1030 secondary school students aged between 11 and 19 years living in the Dominican Republic. The results indicate that emotional intelligence predicts the perception of social support in the samples of both sexes, even though in the female sample the explanatory effect is greater than in the males. It is also observed that the predictive capacity is greater in middle adolescence. These data reinforce the need to develop emotional intelligence.

(c) 2020 Fundación Universitaria Konrad Lorenz. This is an open access article under the CC BYNC-ND license (http://creativecommons.org/licenses/bync-nd/4.0/).

\section{NC-ND license (http://creativecommons.org/licenses/bync-nd/4.0/).}

\section{KEYWORDS}

Emotional intelligence, family support, support from friends, support from significant people, adolescence

\footnotetext{
* Autor para correspondencia.

Correo electrónico: mercedesnancyjimenez@gmail.com
}

https://doi.org/10.14349/sumapsi.2020.v27.n1.3

ISSN 0121-4381, ISSN-E 2145-9797/@ 2020 Fundación Universitaria Konrad Lorenz. Este es un artículo Open Access bajo la licencia CC BY-NCND (http://creativecommons.org/licenses/by-nc-nd/4.0/). 
La inteligencia emocional (IE) representa un concepto multidimensional y complejo que ha sido abordado mediante modelos que enfatizan la posibilidad de considerarla una de las habilidades que componen la inteligencia, con lo cual se amplía la comprensión de esta última. De igual forma, se ha estudiado desde modelos que la proponen como un constructo diferenciado dentro del cual se mezclan habilidades y rasgos de personalidad. A los primeros se les conoce como modelos de habilidad y a los últimos como modelos mixtos. Desde el modelo de habilidad de la IE ésta quedaría definida como aquella "capacidad para monitorear los sentimientos y emociones propias y de los demás; para discriminar entre ellos y para usar esa información como guía de los propios pensamientos y acciones" (Salovey \& Mayer, 1990, p. 189). El modelo de Salovey, Mayer, Goldman, Turvey y Palfai (1995), basado en el modelo propuesto por Salovey y Mayer (1990), es uno de los más utilizados y en el que la IE constituye la suma de tres habilidades: atención, claridad y reparación emocional.

Los modelos mixtos, entre los cuales destaca el modelo de Bar-On (1988; 2006), definen la IE como un conjunto de competencias, habilidades y facilitadores emocionales y sociales relacionados entre sí (Bar-On, 1988). Más que teórica, su fundamentación es empírica (Bar-On, 2006), y el desarrollo del modelo se encuentra asociado al desarrollo de una escala de medida: el Inventario de Cociente Emocional (EQ-i, por sus siglas en inglés Emotional Quotient Inventory, Bar-On, 1997). De acuerdo con esta escala, la IE está compuesta por las siguientes dimensiones: intrapersonal, interpersonal, manejo del estrés, adaptabilidad y estado de ánimo general.

El estudio de la IE en la adolescencia resulta de especial importancia porque esta es una etapa vital en la que los adolescentes se encuentran sometidos a grandes cambios propios, así como a diversas presiones académicas y vitales a edades tempranas (Del Valle, Betegón \& Irurtia, 2018). De este modo un nivel adecuado de IE contribuiría a un uso adecuado de las propias emociones a la hora de resolver los problemas y regular las emociones (Acosta-Prado \& Zárate, 2019) en esta etapa tan cambiante del desarrollo. A este respecto, cabe señalar que pese a que en las últimas décadas se han publicado estudios que apuntan hacia la existencia de diferencias entre varones y mujeres, los resultados son aún contradictorios (Sarrionandia \& Garaigordobil, 2017). Algunos estudios indican que la IE varía en función del sexo y la dimensión que se analiza (Esnaola, Revuelta, Ros \& Sarasa, 2017). Al respecto, se ha encontrado que las mujeres, desde el modelo de habilidades cognitivo-emocionales, han tendido a mostrar mayores puntuaciones en atención, comprensión y percepción emocional (Serrano \& Andreu, 2016), mientras que, en relación con la reparación emocional, los resultados tienden a ser mayores entre los hombres (Gartzia, Artizea, Balluerka \& Barberá, 2012; Serrano \& Andreu, 2016). Por otra parte, desde los modelos mixtos diversas investigaciones han demostrado que las chicas muestran puntuaciones superiores en la dimensión interpersonal (Prieto, Ferrándiz, Ferrando, Sánchez \& Bermejo, 2008; Ugarriza \& Pajares-Del-Águila, 2005). En lo referente a la dimensión intrapersonal, algunos estudios encuentran puntuaciones más elevadas entre los chicos (Ugarriza \& Pajares-Del-Águila, 2005), mientras que otros lo hacen entre las chicas (Valdez, Pérez \& Beltrán, 2010), o no encuentran diferencias significativas en función del sexo (Zavala,
Valadez \& Vargas, 2008). Sí parece, sin embargo, que existen diferencias a favor de los chicos en las dimensiones manejo del estrés y adaptabilidad (Prieto et al., 2008; Sánchez-Núñez et al., 2008; Ugarriza \& Pajares-Del-Águila, 2005).

En relación con la variable edad, resultaría esperable que la IE aumentase con la mayor maduración y experiencia adolescente, algo hacia lo que apuntan los resultados de Bar-On y Parker (2000). No obstante, trabajos longitudinales (10-18 años) no observan cambios notables salvo en dimensiones concretas, como, por ejemplo, el aumento en la adaptabilidad y en la dimensión interpersonal entre la adolescencia inicial y la media (Keefer, Holden \& Parker, 2013), o en el manejo del estrés en las chicas, el cual, al contrario que entre los chicos, entre quienes mostraba una tendencia ascendente, disminuía de forma significativa con la edad (Esnaola et al., 2017). En otras ocasiones, sin embargo, se ha constatado que, a partir de los 13 años, las interacciones sociales y habilidades de adaptación disminuyen entre los chicos, de tal manera que los adolescentes de más edad (16-18 años) presentaban un menor manejo del estrés, teniendo una menor tolerancia y un menor control de impulsos (Ugarriza \& Pajares, 2003).

El apoyo social es un constructo complejo que se ha redefinido de manera progresiva a lo largo de los años. Tal como expresan Azpiazu, Esnaola y Sarasa (2015), una de las definiciones más aceptadas es la que desarrolló Lin (1986), quien lo definió como el conjunto de provisiones instrumentales o expresivas, reales o percibidas, aportadas por la comunidad, las redes sociales y los amigos íntimos, que pueden darse tanto en situaciones cotidianas como en crisis. En la adolescencia esta percepción de apoyo resulta crucial, ya que, independientemente de que exista o no, si el adolescente no la siente, no recurrirá a ella para buscar ayuda (Rowsell, Ciarrochi, Deane \& Heaven, 2016).

Las capacidades emocionales pueden contribuir a la adquisición y la calidad de las habilidades sociales, aumentando también la percepción del apoyo social (Salovey, Bedell, Detweiler \& Mayer, 2000). En este sentido, y más con población adolescente, resulta de especial interés considerar la posible relevancia de la IE en el establecimiento de relaciones interpersonales adecuadas (Salavera-Bordás \& Usán, 2018), debido a la importancia que tiene percibir apoyo social en la adolescencia (Musitu \& Cava, 2003). Especialmente importante resulta, además, más que el apoyo real u objetivo, el sentido de manera subjetiva por el/la adolescente, ya que se considera relevante contar con la percepción de los propios adolescentes sobre los distintos aspectos de su vida (Fernández, Daset \& Castelluccio, 2019).

Por tanto, una variable que resultaría relevante evaluar sería el apoyo social percibido. Estudios que implican ambas variables dentro de la determinación de su asociación con otras (e.g., ajuste psicológico), arrojan evidencias que sugieren que la IE tiene una correlación positiva con el apoyo social percibido (Metaj-Macula, 2017; Zeidner \& Matthews, 2016), así como que la mayor capacidad adolescente para identificar las propias emociones se relaciona con una mayor calidad en las relaciones y una mayor red de apoyo, lo que a su vez promovería dicha habilidad emocional (Rowsell et al., 2016).

Existen también estudios que analizan la capacidad predictiva de la IE sobre el apoyo social. En estos casos, cuando la IE se considera desde el modelo de habilidad, la 
mayoría de los trabajos se han realizado con estudiantes de universidad (Gallagher \& Vella-Brodrick, 2008; Kong, Zhao \& You, 2012; Runcan \& lovu, 2013) y adultos/as mayores (Rey, Extremera \& Sánchez-Álvarez, 2017). De este modo, se observa que las personas con mayores niveles de habilidades de IE son las que perciben un mayor nivel de apoyo social. De igual forma, investigaciones como, por ejemplo, la de Metaj-Macula (2017), con muestra universitaria de Kosovo, señalan que las dimensiones de atención emocional, así como la expresión de emociones, son las que guardan una mayor relación con el apoyo familiar percibido. Otro estudio compuesto por jóvenes mexicanos, con edades entre 18 y 21 años, indicó, por medio de ecuaciones estructurales, que la reparación o regulación emocional influye en una mayor satisfacción en las relaciones personales (Ruvalcaba-Romero, Fernández-Berrocal, Salazar-Estrada \& Gallegos-Guajardo, 2017). Entre adolescentes de Australia se ha observado que las habilidades para identificar emociones predicen el apoyo de los amigos (Claire, Ciarrochi, Heaven \& Deane, 2014).

Desde el modelo mixto, los trabajos encontrados son aún más escasos. Existen investigaciones que indican cómo la IE se relaciona con una mayor conducta prosocial en la adolescencia media, corroborando la importancia de la IE en la competencia social (Frederickson, Petrides \& Simmonds, 2012). Lo anterior, de manera esperable, conduciría a una mejor relación con pares y a un mayor apoyo social. De igual forma, se señala la relevancia de la habilidad intrapersonal e interpersonal a la hora de comprender y manejar las propias emociones y las de los demás, lo que facilitaría un mejor manejo del estrés y promovería relaciones sociales más positivas y placenteras (Cejudo, Rodrigo-Ruiz, López-Delgado \& Losada, 2018). En este mismo sentido apunta la investigación realizada por Di Fabio y Kenny (2012) con un grupo de estudiantes italianos entre los 16 y 20 años de edad, en la que se constató que la IE permite explicar las puntuaciones en el apoyo social percibido, siendo las dimensiones intra e interpersonal las que tienen un mayor efecto explicativo sobre la escala general del apoyo social percibido.

De acuerdo con la investigación previa, cabe destacar que son escasos los estudios que aborden la capacidad predictiva de la inteligencia emocional sobre el apoyo social percibido en la adolescencia en función del sexo y la edad. De igual manera, no se han encontrado trabajos que analicen esta relación entre adolescentes de Latinoamérica.

Por tanto, el objetivo general del estudio es analizar la capacidad predictiva de la IE sobre el apoyo social percibido en una muestra de adolescentes de la República Dominicana. En cuanto a los objetivos específicos serían los siguientes: (1) analizar la capacidad predictiva de la IE sobre el apoyo social percibido en una muestra de adolescentes de la República Dominicana tanto desde el modelo de habilidad como desde un modelo mixto (Bar-On, 2006); (2) analizar la capacidad predictiva de la IE tanto desde el modelo de habilidad (Salovey \& Mayer, 1990) como desde un modelo mixto (Bar-On, 2006) sobre el apoyo social percibido, en una muestra de adolescentes de la República Dominicana en función del sexo; y (3) analizar la capacidad predictiva de la IE tanto desde el modelo de habilidad (Salovey \& Mayer, 1990) como desde un modelo mixto (Bar-On, 2006) sobre el apoyo social percibido en una muestra de adolescentes de la República Dominicana en función de la edad (adolescencia temprana y adolescencia media).
De estos objetivos se derivan las siguientes hipótesis: (1) la IE predice el apoyo social percibido (en cuanto al segundo objetivo, no se hace ninguna hipótesis ya que no se han encontrado estudios que hayan analizado los dos modelos de manera conjunta); (2) la IE tiene mayor capacidad predictiva del apoyo social percibido en las chicas; y (3) la IE tiene mayor capacidad predictiva del apoyo social percibido en la adolescencia media.

\section{Método}

\section{Participantes}

Participaron 1030 estudiantes de educación secundaria con edades entre 11 a 19 años $(M=14.68, D T=1.73)$, de los cuales 579 eran chicas (56.2\%) y 451 chicos (43.8\%) residentes en la República Dominicana. La muestra se dividió en dos subgrupos: adolescencia temprana 11-14 años $(M=13.07, D T=0.90), y$ adolescencia media $15-19$ años $(M=15.98, D T=0.97)$.

\section{Instrumentos}

La inteligencia emocional se midió por medio de dos cuestionarios. El primero representa el modelo de habilidad: Trait Meta Mood Scale (TMMS) de Salovey y Mayer (1990), adaptado y validado al castellano por FernándezBerrocal, Extremera y Ramos (2004). Este instrumento se compone por las subescalas atención emocional, claridad emocional y reparación emocional. La consistencia interna (alpha de Cronbach $\alpha$ ) así como los índices de Omega McDonald $(\omega)$ y fiabilidad compuesta (FC) obtenida en este trabajo para cada escala resultan aceptables: atención $(\alpha=.77, \omega=.74, \mathrm{FC}=.75)$, claridad $(\alpha=.75, \omega=.69, \mathrm{FC}=.69)$ y reparación $(\alpha=.75, \omega=.69, \mathrm{FC}=.70)$.

El segundo cuestionario representa los modelos mixtos: Emotional Quotient Inventory: Youth Version Short (EQ-i:YV-S; Bar-On \& Parker, 2000). Este autoinforme está compuesto por 30 ítems que miden las dimensiones intrapersonal, interpersonal, manejo del estrés y adaptabilidad; con la suma de estas dimensiones se puede conseguir una puntuación estimada de la inteligencia emocional-social general. Asimismo, tiene una quinta escala, impresión positiva, creada con el fin de medir el grado en que los sujetos responden al azar o distorsionan sus respuestas en función del efecto de deseabilidad social. La fiabilidad (alpha de Cronbach, Omega McDonald y fiabilidad compuesta) de las diferentes subescalas resultó la siguiente: intrapersonal $(\alpha=.40, \omega=.56, \mathrm{FC}=.58)$, interpersonal $(\alpha=.60, \omega=.53$, FC =.53), manejo del estrés $(\alpha=.71, \omega=.70, \mathrm{FC}=.70)$, $\mathrm{y}$ adaptabilidad $(\alpha=.71, \omega=.63, \mathrm{FC}=.63)$.

Para evaluar el apoyo social percibido se utilizó el cuestionario Multidimensional Scale of Perceived Social Support (MSPSS) de Zimet, Dahlem, Zimet y Farley (1988), traducido al español y validado en Chile por Arechabala y Miranda (2002). La escala MSPSS evalúa la percepción que tienen las personas del apoyo social informal con el que cuentan y está compuesta por 12 ítems, los cuales recogen información del apoyo social percibido por los individuos en tres áreas: familia, amigos y otros significativos. Esta escala ha mostrado tener una buena fiabilidad y validez en sus tres subescalas: familia, amigos y otros significativos o personas significativas, de modo que se obtuvo, en este estudio, un 
coeficiente alpha de Cronbach de $.80, .84$ y .80 , respectivamente. Los índices de Omega McDonald y fiabilidad compuesta fueron, para el apoyo de la familia .79 - .79; para el apoyo de amistades .71 - .71; y .69 - .69 para apoyo de personas significativas.

\section{Procedimiento}

Se utilizó un estudio descriptivo con enfoque cuantitativo. En primer lugar, se procedió a solicitar permiso a las autoridades del Ministerio de Educación de la República Dominicana. Una vez concedido, se seleccionaron ocho centros educativos de forma aleatoria, seis públicos y dos privados - uno de la zona urbana y siete de la zona rural-, todos los cuales manifestaron durante todo el proceso su disposición a colaborar. En cada caso se expusieron los objetivos de la evaluación y las características de los instrumentos seleccionados. Con el apoyo y la facilitación de la administración de cada centro se convocaron reuniones con las familias del alumnado, a fin de obtener el consentimiento informado de los padres o tutores del alumnado. La aplicación de los instrumentos se realizó de manera presencial, grupal, en las respectivas aulas docentes, recordando la voluntariedad de la participación, el anonimato y el uso confidencial que se realizaría de los datos, así como proporcionando todas las instrucciones y materiales necesarios para facilitar su cumplimentación.

\section{Análisis de datos}

El análisis de los datos se realizó con el software SPSS (IBM Corp. Lanzado en 2015. IBM SPSS Statistics para Windows, Version 23.0. Armonk, NY: IBM Corp.). De igual forma, se recogieron las respuestas de 1330 adolescentes, de las que fue preciso eliminar 300 debido a su cumplimentación incorrecta (más de la mitad de herramientas sin responder o con respuestas extremas, como, por ejemplo, todo el cuestionario cumplimentado con valores 1, 4 o 5). De este modo, los análisis se realizaron con 1030 sujetos. En primer lugar, se procedió a calcular el porcentaje de valores perdidos, obteniéndose un índice máximo de $4.8 \%$, por lo que se consideró aceptable para continuar sin precisar de la imputación de estos (Marshall, Altman, Royston \& Holder, 2010). Posteriormente, a fin de estimar la fiabilidad de las respuestas en los cuestionarios se calculó el coeficiente de consistencia interna alfa de Cronbach, así como el índice Omega McDonald y la fiabilidad compuesta (FC). Para el análisis de la capacidad predictiva de la inteligencia emocional sobre el apoyo social se utilizó el procedimiento de regresión lineal múltiple. La muestra se dividió por sexo (chicas y chicos) y por edades (adolescencia temprana y adolescencia media), ya que, como se ha constatado previamente, algunos estudios han encontrado diferencias significativas en función del sexo y la edad. Como criterio para valorar el tamaño del efecto se utilizó el coeficiente de determinación, tomando en consideración los puntos de corte que sugiere Cohen (1988): $R^{2}=.02$ pequeño; $R^{2}=.13$ moderado; $R^{2}=.26$ grande.

\section{Resultados}

A continuación, se exponen los resultados encontrados en función del sexo de los y las adolescentes participantes.

\section{Resultados obtenidos entre las participantes mujeres}

En primer lugar, se muestran los resultados obtenidos al analizar la capacidad predictiva de las distintas dimensiones de la IE (modelo de habilidad, utilizando el cuestionario TMMS, y el modelo mixto, utilizando el cuestionario EQ-i: YV-S) sobre las diferentes fuentes de apoyo social percibido entre las adolescentes participantes (véase la tabla 1).

Los resultados permiten constatar que la capacidad explicativa de la IE sobre el apoyo social percibido varía en función de la fuente de apoyo y la edad de las adolescentes, destacando el peso de la IE en la predicción en la adolescencia inicial del apoyo percibido de personas significativas y sobre el apoyo de amistades en la adolescencia media.

En concreto, desde el modelo de habilidad, el efecto de la IE sobre el apoyo social de personas significativas resulta moderado en la adolescencia inicial (11-14), siendo pequeño en el resto de las fuentes (familia y amistades). Entre las chicas mayores (15-19) se obtiene un efecto moderado a la hora de explicar el apoyo percibido de las amistades, siendo pequeño en el resto de casos (familia y personas significativas). Además, se constata que, entre las chicas de menor edad (11-14), solo la reparación emocional (sobre apoyo familiar y de personas significativas) y la claridad (sobre apoyo de personas significativas) permitirían predecir mayores puntuaciones de apoyo social. Entre las chicas mayores (15-19) serían mayores niveles de claridad y reparación emocional los que permitirían predecir mayor apoyo social percibido por parte de la familia, las amistades y de personas significativas.

Considerando la IE desde un modelo mixto, de nuevo el mayor efecto (de tamaño moderado) se constata sobre el apoyo percibido de personas significativas en la adolescencia inicial. Sobre el resto de fuentes el efecto es pequeño. En el caso de la adolescencia media, el mayor efecto de la IE se observa sobre el apoyo percibido de amistades, si bien este resulta -como en las demás fuentes de apoyopequeño.

Tanto entre las adolescentes jóvenes (11-14) como entre las mayores (15-19 años) la dimensión intrapersonal explicaría mayor apoyo social percibido (de amistades en el primer caso, de amistades y familia en el segundo). La dimensión interpersonal explicaría mayor apoyo percibido por parte de las tres fuentes entre las adolescentes de ambos tramos de edad, si bien en distinta medida en cada caso; personas significativas, amistades y, en menor medida, familiar entre las chicas mayores (15-19), y personas significativas, familia y amistades entre las jóvenes.

\section{Resultados entre los adolescentes hombres}

En la tabla 2 se muestran los resultados obtenidos al analizar la capacidad predictiva de las distintas dimensiones de la IE, en sus dos modelos, sobre las diferentes fuentes de apoyo social percibido en la muestra masculina (véase la tabla 2).

Entre los chicos, la IE, considerada desde sus dos modelos, revela menor capacidad explicativa sobre el apoyo social, siendo, en todo caso, el efecto de dicha variable pequeño sobre cualquiera de las tres fuentes de apoyo. Especialmente, entre los chicos de menor edad (11-14), desde el modelo de habilidad solo la dimensión de reparación permite explicar mayor percepción de apoyo familiar, 
Tabla 1 Capacidad predictiva de la IE sobre el apoyo social de las chicas

\begin{tabular}{|c|c|c|c|c|c|c|c|c|c|c|c|c|c|}
\hline & & Apoyo & e pers & has sigr & cativas & & Apoyc & amilia & & & pyo de & s ami & \\
\hline \multirow{12}{*}{ 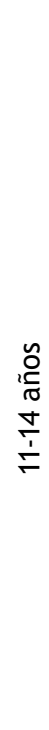 } & $\begin{array}{l}\text { Inteligencia } \\
\text { emocional }\end{array}$ & $R^{2}$ & $\beta$ & $T$ & $p$ & $R^{2}$ & $\beta$ & $t$ & $p$ & $R^{2}$ & $\beta$ & $T$ & $p$ \\
\hline & TMMS & & & & & & & & & & & & \\
\hline & Constante & & - & 8.05 & .000 & & - & 8.85 & .000 & & - & 6.83 & .000 \\
\hline & Atención & 110 & .293 & 3.61 & .000 & 096 & .042 & .505 & .615 & (2) & .093 & 1.07 & .285 \\
\hline & Claridad & טדו. & -.012 & -.127 & .899 & ט & .070 & .744 & .458 & .020 & .034 & .351 & .726 \\
\hline & Reparación & & .190 & 2.22 & .028 & & .271 & 3.09 & .002 & & .114 & 1.25 & .214 \\
\hline & EQ-i:YV-S & & & & & & & & & & & & \\
\hline & Constante & & - & 4.95 & .000 & & - & 5.90 & .000 & & .000 & 2.07 & .040 \\
\hline & Intrapersonal & & .054 & .763 & .447 & & .057 & .750 & .454 & & .201 & 2.71 & .008 \\
\hline & Interpersonal & .181 & .451 & 5.62 & .000 & .047 & .255 & 2.95 & .004 & .091 & .221 & 2.61 & .010 \\
\hline & Manejo estrés & & -.010 & -.143 & .887 & & .018 & .233 & .816 & & .113 & 1.53 & .128 \\
\hline & Adaptabilidad & & -.017 & -.213 & .831 & & .007 & .077 & .939 & & .062 & .728 & .468 \\
\hline \multirow{12}{*}{ 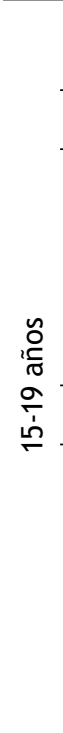 } & $\begin{array}{l}\text { Inteligencia } \\
\text { Emocional }\end{array}$ & & & & & & & & & & & & \\
\hline & TMMS & & & & & & & & & & & & \\
\hline & Constante & \multirow{4}{*}{.095} & - & 9.69 & .000 & \multirow{4}{*}{.078} & - & 7.35 & .000 & \multirow{4}{*}{.140} & - & 6.75 & .000 \\
\hline & Atención & & .043 & .725 & .469 & & -.079 & -1.34 & .181 & & .085 & 1.49 & .138 \\
\hline & Claridad & & .227 & 3.55 & .000 & & .148 & 2.30 & .022 & & .221 & 3.55 & .000 \\
\hline & Reparación & & .113 & 1.83 & .043 & & .218 & 3.50 & .001 & & .171 & 2.85 & .005 \\
\hline & \multicolumn{13}{|l|}{ EQ-i:YV-S } \\
\hline & Constante & & - & 5.73 & .000 & & - & 3.65 & .000 & & - & 3.48 & .001 \\
\hline & Intrapersonal & & .076 & 1.41 & .159 & & .150 & 2.71 & .007 & & .214 & 4.01 & .000 \\
\hline & Interpersonal & .093 & .293 & 4.67 & .000 & .043 & .188 & 2.92 & .004 & .104 & .230 & 3.68 & .000 \\
\hline & Manejo estrés & & -.026 & -.485 & .628 & & .070 & 1.26 & .207 & & .016 & .295 & .768 \\
\hline & Adaptabilidad & & .020 & .325 & .745 & & -.059 & -.914 & .361 & & .034 & .547 & .585 \\
\hline
\end{tabular}

Nota. $\beta$ = Coeficiente beta; $\mathrm{R}^{2}=$ cuadrado corregido; $\mathrm{t}=$ estadísticos $t$.

mientras que desde el modelo mixto únicamente la dimensión interpersonal y el manejo del estrés explicarían mayor apoyo percibido (por parte de familia y personas significativas en el primer caso, y de amigos en el segundo).

Entre los adolescentes de mayor edad (15 y 19 años), además de la reparación emocional (sobre personas significativas), la claridad predice mayor apoyo familiar percibido. Desde el modelo mixto, como entre los chicos de menor edad, de nuevo son solo dos, la dimensión interpersonal (sobre personas significativas y amistades) y el manejo del estrés (sobre el apoyo familiar), las que permiten explicar el apoyo social percibido.

\section{Discusión}

El objetivo general del estudio era analizar la capacidad predictiva de la IE sobre el apoyo social percibido en una muestra de adolescentes de la República Dominicana. En este sentido, es posible concluir que, en general, la IE predice el apoyo social percibido confirmando (en general o parcialmente) la primera hipótesis.

En cuanto al primer objetivo específico (analizar la capacidad predictiva de la IE sobre el apoyo social percibido en una muestra de adolescentes de la República Dominicana tanto desde el modelo de habilidad como desde un modelo mixto), los resultados encontrados señalan que ambos modelos permiten explicar el apoyo social percibido tanto de chicos como de chicas, si bien la capacidad predictiva alcanzada resulta moderada-baja, lo que podría estar indicando que la relación entre IE y apoyo social es más compleja y se encuentra mediada por variables no consideradas en estos modelos.

El tercer objetivo específico trataba de analizar la capacidad predictiva de la IE tanto desde el modelo de habilidad 
Tabla 2 Capacidad predictiva de la IE sobre el apoyo social de los chicos

\begin{tabular}{|c|c|c|c|c|c|c|c|c|c|c|c|c|c|}
\hline & & Apoyo & perso & as sign & cativa: & & Apoy & familic & & & poyo d & os ami & \\
\hline \multirow{12}{*}{ 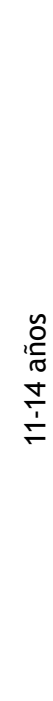 } & Inteligencia emocional & $\mathrm{R}^{2}$ & $\beta$ & $T$ & $p$ & $\mathrm{R}^{2}$ & $\beta$ & $t$ & $p$ & $\mathrm{R}^{2}$ & $\beta$ & $T$ & $p$ \\
\hline & TMMS & & & & & & & & & & & & \\
\hline & Constante & \multirow{4}{*}{.005} & - & 5.81 & .000 & \multirow{4}{*}{.090} & & 6.76 & .000 & \multirow{4}{*}{.009} & - & 5.96 & .000 \\
\hline & Atención & & -.021 & -.175 & .862 & & -.068 & -.594 & .554 & & .093 & .783 & .436 \\
\hline & Claridad & & .105 & .810 & .420 & & .159 & 1.29 & .201 & & .151 & 1.17 & .245 \\
\hline & Reparación & & .114 & .969 & .335 & & .260 & 2.32 & .023 & & -.049 & -.421 & .675 \\
\hline & EQ-i:YV-S & & & & & & & & & & & & \\
\hline & Constante & & - & 1.26 & .212 & & - & 2.84 & .006 & & - & 1.35 & .179 \\
\hline & Intrapersonal & & .054 & .545 & .587 & & -.050 & -.512 & .610 & & -.068 & -.691 & .491 \\
\hline & Interpersonal & .067 & .326 & 2.74 & .007 & .110 & .356 & 3.06 & .003 & .077 & .231 & 1.95 & .054 \\
\hline & Manejo estrés & & .176 & 1.66 & .100 & & .076 & .738 & .462 & & .211 & 2.00 & .048 \\
\hline & Adaptabilidad & & .004 & .028 & .977 & & .077 & .642 & .523 & & .174 & 1.41 & .161 \\
\hline \multirow{12}{*}{ 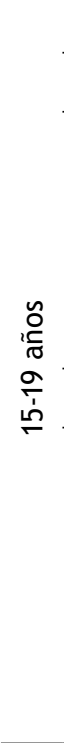 } & Inteligencia Emocional & & & & & & & & & & & & \\
\hline & TMMS & & & & & & & & & & & & \\
\hline & Constante & \multirow{4}{*}{.058} & - & 8.37 & .000 & \multirow{4}{*}{.026} & - & 9.38 & .000 & \multirow{4}{*}{.004} & - & 8.69 & .000 \\
\hline & Atención & & -.052 & -.765 & .445 & & -.134 & -1.92 & .056 & & -.001 & -.015 & .988 \\
\hline & Claridad & & .106 & 1.49 & .138 & & .142 & 1.97 & .050 & & .071 & .965 & .336 \\
\hline & Reparación & & .218 & 3.21 & .001 & & .117 & 1.70 & .091 & & .076 & 1.08 & .280 \\
\hline & \multicolumn{13}{|l|}{ EQ-i:YV-S } \\
\hline & Constante & \multirow{5}{*}{.034} & - & 4.12 & .000 & \multirow{5}{*}{.010} & - & 4.78 & .000 & \multirow{5}{*}{.024} & - & 3.53 & .000 \\
\hline & Intrapersonal & & -.008 & -.138 & .891 & & -.029 & -.478 & .633 & & .085 & 1.39 & .165 \\
\hline & Interpersonal & & .176 & 2.60 & .010 & & .016 & .234 & .815 & & .178 & 2.63 & .009 \\
\hline & Manejo estrés & & .103 & 1.65 & .100 & & .158 & 2.50 & .013 & & .085 & 1.36 & .177 \\
\hline & Adaptabilidad & & .075 & 1.09 & .279 & & .067 & .946 & .345 & & -.032 & -.461 & .645 \\
\hline
\end{tabular}

Nota. $\beta$ = Coeficiente beta; $\mathrm{R}^{2}=$ cuadrado corregido; $\mathrm{t}=$ estadísticos $t$.

(Salovey \& Mayer, 1990) como desde un modelo mixto (Bar-On, 2006) sobre el apoyo social percibido en una muestra de adolescentes de la República Dominicana en función del sexo. Los resultados han demostrado, por una parte, que la IE predice la percepción del apoyo social tanto en la muestra de chicos como de chicas, aun cuando en esta última la capacidad predictiva es mayor que entre los varones, lo que confirma la segunda hipótesis. Esto podría explicarse considerando que, según algunos estudios, las chicas suelen identificar un número mayor de emociones y presentan una mayor probabilidad de identificar conductas interpersonales empáticas (Sánchez-Núñez et al., 2008; Reina \& Oliva, 2015), lo cual tiene efectos positivos en la disponibilidad del apoyo social y en la calidad de las relaciones sociales (Salovey et al., 2000). De igual manera, estas diferencias podrían ser consecuencia de que el proceso de socialización, en el caso de las chicas, suele estar más centrado en la expresividad, afecto y vinculación a otros, mientras que para los chicos suele estar más enfocado en la autonomía, la instrumentalidad y la independencia (Musitu \& Cava, 2003).

El cuarto objetivo específico trataba de analizar la capacidad predictiva de la IE, tanto desde el modelo de habilidad como desde un modelo mixto sobre el apoyo social percibido, en una muestra de adolescentes de la República Dominicana en función de la edad (adolescencia temprana y adolescencia media). La IE ha permitido explicar en mayor medida el apoyo social percibido por las mujeres entre 15 y 19 años, grupo en el cual se ha observado, desde la perspectiva del modelo de habilidad, que la dimensión de reparación emocional predice de forma positiva el apoyo social percibido en sus tres componentes, con un mayor impacto en el apoyo familiar, en línea con estudios previos en los que, entre jóvenes universitarios (18-21), la capacidad de regulación se asociaba a una mayor satisfacción en las 
relaciones personales (Ruvalcaba-Romero et al., 2017). En el caso de los chicos de la misma edad, la reparación emocional solo predice el apoyo de las personas significativas. Sobre esto conviene tener en cuenta que algunos estudios señalan que el comienzo de la pubertad supone una disminución de la habilidad de comprensión y regulación de las emociones (Somerville, Jones \& Casey, 2010), cuestión que va cambiando a medida que el adolescente adquiere una mayor edad (Keefer et al., 2013), lo cual podría explicar por qué la capacidad predictiva de la reparación sobre el apoyo social aumenta también con la edad. También otros estudios indican que la habilidad para regular emociones está asociada con la calidad autopercibida de las relaciones interpersonales (Mestre \& Fernández-Berrocal, 2009).

Por otra parte, en el caso de las chicas, la claridad emocional y la reparación emocional han aparecido como predictores del apoyo de las tres fuentes en la adolescencia media. En la adolescencia temprana, sin embargo, solo la reparación explica el apoyo de la familia y de personas significativas (apoyo, el de las personas significativas, que también puede explicarse a través de la atención a las propias emociones), mientras en los chicos de la misma etapa la reparación emocional es la única dimensión que predice el apoyo de la familia.

Estos resultados apuntarían en la misma línea de investigaciones previas en las que se ha observado que los y las jóvenes con mayores niveles de habilidades de IE son quienes perciben un mayor nivel de apoyo social (Gallagher \& Vella-Brodrick, 2008; Kong et al., 2012; Runcan \& lovu, 2013), así como que comprender y regular bien los estados emocionales son las habilidades que permiten predecir en mayor medida el apoyo social percibido (Montes-Berges \& Augusto, 2007).

Es posible que en la adolescencia temprana las chicas que prestan más atención a sus emociones tiendan a buscar más apoyo de personas significativas, percibiendo así mayor apoyo social, mientras que con la madurez esta búsqueda de apoyo se produzca solo en el caso de observar determinados estados emocionales, lo que coincidiría con observaciones previas en las que una mayor capacidad adolescente para identificar las propias emociones se relaciona con una mayor calidad en las relaciones y una mayor red de apoyo (Claire et al., 2014; Rowsell et al., 2016).

Al mismo tiempo, con la progresiva mejor gestión y reparación emocional adquirida con la edad, es posible que precisamente aquellas chicas que son capaces de reparar sus propias emociones (ante conflictos, o para encontrarse con los demás), sean quienes, por dicha razón, perciban también un mayor apoyo de su red social. Quizá el que las chicas difieran de los chicos en esta franja de edad dependa de la maduración más temprana del sistema nervioso central de ellas, implicado en la regulación emocional (De Bellis et al., 2001), así como del efecto del contexto (Clarke, Marks \& Lykins, 2016), debido a la socialización diferencial que educa a las mujeres hacia la relación y el cuidado de las interacciones personales (Gartzia et al., 2012).

Por otra parte, en cuanto al modelo mixto, se ha constatado -en línea con estudios previos (Cejudo et al., 2018; Di Fabio \& Kenny, 2012)-, la importancia de las dimensiones inter e intrapersonal en la percepción de apoyo social. De hecho, en el presente trabajo se ha observado que la dimensión intrapersonal predice de forma positiva el apoyo social percibido de los amigos y la familia en las mujeres de 15 a 19 años, mientras que la dimensión interpersonal predice el apoyo familiar, el apoyo de los amigos y el de personas significativas en las dos franjas de edad. Sin embargo, en el caso de los chicos, si bien la dimensión interpersonal permite explicar las puntuaciones de apoyo de personas significativas a lo largo de toda la adolescencia, en la adolescencia temprana explicaría, además, el apoyo de los amigos, mientras que en la adolescencia media explicaría el familiar. Entre los chicos, además, la dimensión de manejo del estrés - que no ha sido significativa en el caso de las chicas- permite explicar el apoyo de los amigos en la adolescencia temprana y de la familia en la adolescencia media. Esto podría deberse a que una mejor gestión del propio estrés podría contribuir a reducir el nivel de conflicto intrafamiliar propio de la etapa adolescente y, por consiguiente, a sentir un mayor apoyo de la familia (Sanz, 2018). Todos estos resultados confirman la tercera hipótesis según la cual la IE tendría mayor capacidad predictiva del apoyo social percibido en la adolescencia media.

No obstante, es preciso señalar que este trabajo no se encuentra exento de limitaciones que deben tenerse en consideración. En primer lugar, su naturaleza transversal hace que no puedan extraerse conclusiones respecto a la posible evolución o desarrollo de la IE a lo largo de la adolescencia, por lo que sería preciso, en futuros estudios, considerar un diseño longitudinal que permita contrastar estos resultados. Una segunda limitación a reseñar es el hecho de que no se aplicaron escalas de deseabilidad social que permitieran determinar la influencia de este elemento en las respuestas de los participantes, ni de infrecuencia de respuesta para la verificación de la consistencia de estas. Por otra parte, resulta importante señalar que no se han tomado en cuenta variables que podrían estar condicionando los resultados, tales como los estudios previos de los progenitores, su nivel socioeconómico, el tipo o estructura de familia y sus aspectos propios de los y las adolescentes (autoconcepto, personalidad, $\mathrm{Cl}$, posible historial de problemas previos de salud mental, etc.). Además, el limitado tamaño de efecto obtenido, especialmente en el caso de los chicos, parece estar apuntado al hecho de que no se han considerado, en el modelo, variables que pueden ser relevantes a la hora de explicar su percepción de apoyo social. Esto debería considerarse en futuras investigaciones, a fin de profundizar en la complejidad de las relaciones entre el desarrollo de la IE y la percepción del apoyo social entre adolescentes. En este sentido, sería enriquecedor hacerlo mediante el uso de ecuaciones estructurales que permitan examinar posibles efectos mediadores y variables latentes, así como relaciones de dependencia entre las variables de forma simultánea. Otra limitación a tener en cuenta ha sido la baja consistencia interna obtenida en las dimensiones interpersonal e intrapersonal, por lo que la interpretación de los resultados relativos a estas subescalas debería hacerse con cautela. De igual manera debería obrarse a la hora de generalizar los resultados aquí mostrados, ya que han sido obtenidos con unas herramientas concretas que, en futuras investigaciones, sería preciso validar y constatar su equivalencia en población dominicana. Cabe señalar que, además, si bien los resultados se obtienen a partir de un número relevante de adolescentes, toda muestra resulta siempre limitada y el contexto se circunscribe a la República 
Dominicana, siendo escasos los estudios con participantes latinoamericanos y poco frecuentes las investigaciones que se centren en el mismo objeto de estudio (la capacidad predictiva de la IE sobre el apoyo social percibido en adolescentes), lo que dificulta la comparación y generalización de estos resultados.

A pesar de las limitaciones reseñadas, la confirmación de que la IE permite predecir el apoyo social constituye un dato importante, pues podría significar que fomentar el desarrollo de la IE en adolescentes ayudaría a fortalecer la percepción positiva que estos tengan de sus relaciones sociales e interpersonales. Esto podría tener un efecto positivo en el trabajo con adolescentes que presenten algún tipo de problema en su forma de relacionarse con la sociedad, teniendo en cuenta que la valoración subjetiva de la calidad del apoyo social refuerza la autoestima y tiene efectos positivos en el bienestar y en el manejo del estrés de los adolescentes, así como en su forma de relacionarse con su entorno (Chavarría \& Barra, 2013). De igual manera, los datos separados por sexo y edad podrían facilitar que los profesionales encuentren qué dimensiones se pueden trabajar en mayor medida con los y las adolescentes de distintas edades, a fin de incidir de manera positiva en su percepción del apoyo social.

\section{Referencias}

Acosta-Prado, J. C., \& Zárate, R. A. (2019). Validation of the Wong and Law Emotional Intelligence Scale for Chilean managers. Suma Psicológica, 26(2), 110-118. https://doi.org/10.14349/ sumapsi.2019.v26.n2.7

Arechabala, M. C., \& Miranda, C. (2002). Validación de una escala de apoyo social percibido en un grupo de adultos mayores adscritos a un programa de hipertensión de la región metropolitana. Ciencia y Enfermería, 8(1), 49-55.

Azpiazu, L., Esnaola, I., \& Sarasa, M. (2015). Capacidad predictiva del apoyo social en la inteligencia emocional de adolescentes. European Journal of Education and Psychology, 8(1), 23-29.

Bar-On, R. (1988). The development of a concept of psychological well-being (Tesis de doctorado). Rhodes University, Sudáfrica.

Bar-On, R. (1997). The Bar-On Emotional Quotient Inventory (EQ-i): technical manual. Toronto: Multi-Health Systems.

Bar-On, R. (2006). The Bar-On model of emotional-social intelligence (ESI). Psicothema, 18,13-25.

Bar-On, R., \& Parker, J. (2000). The Bar-On Emotional Quotient Inventory: youth version (EQ-i: YV) technical manual (translated to Spanish by Caraballo, C. M. \& Villegas, O.). Toronto: Multi-HealthSystems.

Cejudo, J., Rodrigo-Ruiz, D., López-Delgado, M. L., \& Losada, L. (2018). Emotional intelligence and its relationship with levels of social anxiety and stress in adolescents. International Journal of Environmental Research and Public Health, 15(6), 1073-1084. https://doi.org/10.3390/ijerph15061073

Chavarría, M. P., \& Barra, E. (2013). Satisfacción vital en adolescentes: relación con la autoeficacia y el apoyo social percibido. Terapia Psicológica, 32(1), 41-46. https://doi.org/10.4067/ S0718-48082014000100004

Claire, H. R., Ciarrochi, J., Heaven, P. C., \& Deane, F. P. (2014). The role of emotion identification skill in the formation of male and female friendships: a longitudinal study. Journal of Adolescence, 37(2), 103-111.

Clarke, M. J., Marks, A. D., \& Lykins, A. D. (2016). Bridging the gap: the effect of gender normativity on differences in empathy and emotional intelligence. Journal of Gender Studies, 25(5), 522539. https://doi.org/10.1080/09589236.2015.1049246
Cohen, J. (1988). Statistical power analysis for the behavioral sciences. Hillsdale, NJ: Erlbaum.

De Bellis, M. D., Keshavan, M. S., Beers, S. R., Hall, J., Frustaci, K., Masalehdan, A., Noll, J., \& Boring, A. M. (2001). Sex differences in brain maturation during childhood and adolescence. Cerebral Cortex, 11, 552-557.

Del Valle, M., Betegón, E., \& Irurtia, M. J. (2018). Efecto del uso de estrategias cognitivas de regulación emocional sobre la ansiedad en adolescentes españoles. Suma Psicológica, 25(2), 153161. https://doi.org/10.14349/sumapsi.2018.v25.n2.7

Di Fabio, A., \& Kenny, M. E. (2012). Emotional intelligence and perceived support among Italian high school students. Journal of Career Development, 39, 459-473.

Esnaola, I., Revuelta, L., Ros, I., \& Sarasa, M. (2017). The development of emotional intelligence in adolescence. Anales de Psicología, 33(2), 211-217.

Fernández, M. E., Daset, L. R., \& Castelluccio, L. (2019). Perfil de bienestar psicológico subjetivo en adolescentes uruguayos. Suma Psicológica, 26(2), 103-109. https://doi.org/10.14349/ sumapsi.2019.v26.n2.6

Fernández-Berrocal, P., Extremera, N., \& Ramos, N. (2004). Validity and reliability of the Spanish modified version of the Trait Meta-Mood Scale. Psychological Reports, 94(3), 751-755. https:// doi.org/10.2466/pr0.94.3.751-755

Frederickson, N., Petrides, K. V., \& Simmonds, E. (2012). Trait emotional intelligence as a predictor of socioemotional outcomes in early adolescence. Personality and Individual Differences, 52, 323-328. https://doi.org/10.1016/j.paid.2011.10.034

Gallagher, E. N., \& Vella-Brodrick, D. A. (2008). Social support and emotional intelligence as predictors of subjective well-being. Personality and Individual Differences, 44(7), 1551-1561. https://doi.org/10.1016/j.paid.2008.01.011

Gartzia, L., Aritzeta, A., Balluerka, N., \& Barberá, E. (2012). Inteligencia emocional y género: más allá de las diferencias sexuales. Anales de Psicología, 28(2), 567-575. https://doi.org/10.6018/ analesps.28.2.124111

Keefer, K. V., Holden, R. R., \& Parker, J. D. A. (2013). Longitudinal assessment of trait emotional intelligence. Measurement invariance and construct continuity from late childhood to adolescence. Psychological Assessment, 24(4), 1255-1272.

Kong, F., Zhao, J., \& You, X. (2012). Emotional intelligence and life satisfaction in Chinese university students: the mediating role of self-esteem and social support. Personality and Individual Differences, 53(8), 1039-1043.

Lin, N. (1986). Conceptualizing social support. En N. Lin, A. Dean \& W. Ensel (Eds.), Social Support, life events, and depression (pp. 17-30). Nueva York: Academic Press.

Marshall, A., Altman, D. G., Royston, P., \& Holder, R. L. (2010). Comparison of techniques for handling missing covariate data within prognostic modelling studies: a simulation study. BMC Medical Research Methodology, 10(7), 1-16. https://doi. org/10.1186/1471-2288-10-7

Mestre, J. M., \& Fernández-Berrocal, P. (2009). Manual de inteligencia emocional. Madrid: Ediciones Pirámide.

Metaj-Macula, A. (2017). The relationship between emotional intelligence and perceived social support. Journal of Educational and Social Research, 7(1), 168-172.

Montes-Berges, B., \& Augusto, J. M. (2007). Exploring the relationship between perceived emotional intelligence, coping, social support and mental health in nursing students. Journal of Psychiatric and Mental Health Nursing, 14(2), 163-171.

Musitu, G., \& Cava, M. J. (2003). El apoyo social en el ajuste de los adolescentes. Intervención Psicosocial, 12(2), 179-192.

Prieto, M. D., Ferrándiz, C., Ferrando, M., Sánchez, C., \& Bermejo, M. R. (2008). Inteligencia emocional y alta habilidad. Revista Española de Pedagogía, 240, 241-260.

Reina, M. D. C., \& Oliva, A. (2015). De la competencia emocional a la autoestima y satisfacción vital en adolescentes. Psicología Conductual, 23(2), 345-359. 
Rey, L., Extremera, N., \& Sánchez-Álvarez, N. (2017). Clarifying the links between perceived emotional intelligence and well-being in older people: pathways through perceived social support from family and friends. Applied Research in Quality of Life, 14(1), 221-235. https://doi.org/10.1007/s11482-017-9588-6

Rowsell, H. C., Ciarrochi, J., Deane, F. P., \& Heaven, P. C. (2016). Emotion identification skill and social support during adolescence: a three-year longitudinal study. Journal of Research on Adolescence, 26(1), 115-125. https://doi.org/10.1111/jora.12175

Runcan, P. L., \& lovu, M. B. (2013). Emotional intelligence and life satisfaction in Romanian university students: the mediating role of self-esteem and social support. Revista de Cercetare si Interventie Sociala, 40, 137-148.

Ruvalcaba-Romero, N. A., Fernández-Berrocal, P., Salazar-Estrada, J. G., \& Gallegos-Guajardo, J. (2017). Positive emotions, self-esteem, interpersonal relationships and social support as mediators between emotional intelligence and life satisfaction. Journal of Behavior, Health and Social Issues, 9(1), 1-6. https:// doi.org/10.1016/j.jbhsi.2017.08.001

Salavera-Bordás, C., \& Usán, P. (2018). Uso del humor e inteligencia emocional en estudiantes de Secundaria. Revista Electrónica Interuniversitaria de Formación del Profesorado, 21(3), 109-122.

Salovey, P., Bedell, B. T., Detweiler, J. B., \& Mayer, J. D. (2000). Current directions in emotional intelligence research. En $M$. Lewis \& J. M. Haviland-Jones (Eds.), Handbook of emotions (pp. 504520). Nueva York: Guilford Press.

Salovey, P., \& Mayer, J. D. (1990). Emotional intelligence. Imagination, Cognition and Personality, 9(3), 185-211.

Salovey, P., Mayer, J. D., Goldman, S. L., Turvey, C., \& Palfai, T. P. (1995). Emotional attention, clarity, and repair: exploring emotional intelligence using the Trait Meta-Mood Scale. En J. W. Pennebaker (Ed.), Emotion, disclosure, and health (pp. 125154). Washington: American Psychological Association.

Sánchez-Núñez, M., Fernández-Berrocal, P., Montañés, J., \& Latorre, J. M. (2008). Does emotional intelligence depend on gender? The socialization of emotional competencies in men and women and its implications. Electronic Journal of Research in Educational Psychology, 6(2), 455-474.
Sanz, D. M. (2018). Niveles de actividad física y apoyo social percibido del alumnado adolescente de la provincia de Soria (Tesis de doctorado). Universidad Complutense de Madrid, España.

Sarrionandia, A., \& Garaigordobil, M. (2017). Efectos de un programa de inteligencia emocional en factores socioemocionales y síntomas psicosomáticos. Revista Latinoamericana de Psicología, 49(2), 110-118. https://doi.org/10.1016/j.rlp.2015.12.001

Serrano, C., \& Andreu, Y. (2016). Perceived emotional intelligence, subjective well-being, perceived stress, engagement and academic achievement of adolescents. Revista de Psicodidáctica, 21(2), 357-374. https://doi.org/10.1387/RevPsicodidact.14887

Somerville, L. H., Jones, R. M., \& Casey, B. J. (2010). A time of change: behavioral and neural correlates of adolescent sensitivity to appetitive and aversive environmental cues. Brain and Cognition, 72(1), 124-133.

Ugarriza, N., \& Pajares, L. (2003). Adaptación y estandarización del inventario de inteligencia emocional de Bar-On ICE: en niños y adolescentes. Perú: Libro amigo.

Ugarriza, N., \& Pajares-Del-Águila, L. (2005). La evaluación de la inteligencia emocional a través del inventario de Bar-On ICE: NA, en una muestra de niños y adolescentes. Persona, 8, 11-58.

Valdez, M. D., Pérez, L., \& Beltrán J. (2010). La inteligencia emocional de los adolescentes talentosos. Faísca, 15(17), 2-17.

Zavala, M. A., Valadez, M. D., \& Vargas, M. C. (2008). Emotional intelligence and social skills in adolescents with high social acceptance. Electronic Journal of Research in Educational Psychology, 6(2), 319-338.

Zeidner, M., \& Matthews, G. (2016). Ability emotional intelligence and mental health: social support as a mediator. Personality and Individual Differences, 99, 196-199.

Zimet, G. D., Dahlem, N. W., Zimet, S. G., \& Farley, G. K. (1988). The multidimensional scale of perceived social support. Journal of Personality Assessment, 52(1), 30-41. https://doi.org/10.1207/ s15327752jpa5201_2 